\title{
Purging and isolating pluripotent cells, "sweet" dreams become true?
}

\author{
Ignacio Sancho-Martinez ${ }^{1}$, Emmanuel Nivet ${ }^{1}$, Juan Carlos Izpisua Belmonte ${ }^{1,2}$ \\ ${ }^{1}$ Gene Expression Laboratory, The Salk Institute for Biological Studies, 10010 North Torrey Pines Road, La Jolla, CA 92037, USA; \\ ${ }^{2}$ Center for Regenerative Medicine in Barcelona, Dr Aiguader 88, 08003 Barcelona, Spain \\ Cell Research (2011) 21:1526-1527. doi:10.1038/cr.2011.163; published online 25 October 2011
}

The formation of an adult organism could be viewed as a hierarchical process in which the initial totipotent cell, the zygote, progressively loses "potency" by differentiating into pluripotent, multipotent and unipotent states until the final terminally differentiated cells comprising tissues and organs are derived. Such a unidirectional concept trembled when four transcription factors were shown to "revert" the identity of differentiated somatic cells and reprogram them into induced pluripotent stem cells (iPSCs) [1]. These findings rapidly fed clinical expectations for regenerative medicine purposes as well as for disease modeling and drug discovery studies [2]. Though this discovery represented a promising breakthrough, the technologies applied raised several concerns, as thoroughly discussed in other reports [3]. Importantly, and regardless of the methodology used for reprogramming, the lack of efficient differentiation protocols opened the possibility that mixed populations could interfere during in vitro studies. Furthermore, experimental assessment of pluripotency involves the formation of benign tumors, called teratomas. Thus, residual pluripotent stem cells need to be eliminated prior to transplantation

Correspondence: Juan Carlos Izpisua Belmonte E-mail: belmonte@salk.edu, izpisua@cmrb.eu in order to reduce the risk of malignant transformations [3]. Up to date, efficient selection of residual pluripotent stem cells (PSCs) relied on the use of antibodies recognizing known pluripotency-related surface markers as well as tissue-specific fluorescence reporters combined with sorting procedures [4]. Additionally, suicide gene strategies have also been described [5]. Yet, such methods can lead to undesired cellular modifications due to antibody binding and/or alteration of the host genome.

The concept that surface glycans might represent a more convenient and reliable method for defining cell identity has been previously suggested [6]. Indeed, a large set of glycans is strategically located on the cell surface and the overall profile is believed to faithfully reflect cellular alterations, such as differentiation and even malignancy. After all, around $50 \%$ of the total pool of eukaryotic proteins undergo glycosylation to some extent [7]. Up to date, most of the antibodies and markers used for stem cell biology rely on the identification of glycoproteins such as Tra1-60, Tra1-81 and SSEA3/4. Yet, some carbohydrate structures have proven to be poor antigens. Furthermore, even though antibodies can be generated, potential activation of downstream pathways, crosslinking of proteins, as well as the inherent affinity of antibody-antigen complexes remain as some of the major caveats for antibody-based procedures. Considering certain living cell applications after sorting, the permanent binding of antibodies might not only disturb experimental results but also brings the possibility for immune reaction in vivo. Thus, antibody-based strategies for sorting might not be the preferred choice for the researcher, depending on the experiments planned after selection. More importantly, antibodies recognizing native conformations of proteins, specifically in stem cell research, tend to show species-dependent specificity to varying degrees. Thus, it is not uncommon for different laboratories working on different models to have different antibodies in order to recognize the same protein depending on the species, which can be a concern, and an extra expense, that could be avoided by recognition of surface glycans.

Interestingly, a recent report from the Loring laboratory, convincingly established glycan profiling and lectinbased selection procedures as a reliable alternate method for the discrimination of PSCs from differentiated cultures [8]. In their report, Wang et al. described the expression levels of glycoproteins present both on the cell surface and in the intracellular fraction of pluripotent cells and compared them to those observed in differentiated and tumor cells. The authors made use of an "old" concept, the binding of specific lectins 
to glycans, recently expanded into microarray technologies [6]. Indeed, lectin microarrays allowed for the elucidation of cell-specific glycan profiles by multiplexing lectin-glycan interactions and thus, establishing a proteomic profile similar to conventional microarray RNA profiling. Two previous studies have demonstrated the feasibility of discriminating human PSCs from differentiated cells $[9,10]$. Yet, it was not clear until now whether the same approach would suffice for the negative or positive selection of hPSCs in mixed populations, that is the separation of residual pluripotent cells from differentiated cultures. By using lectin binding-based arrays, the authors were able to identify a number of consistently over- and under-represented glycoproteins defining cellular identity. Those molecules present on the cell surface were further studied for their capacity to precisely distinguish between pluripotent and non-pluripotent cells with the goal of establishing a way to select and isolate specific cell populations. Specifically, UEA-1 was proven to strongly correlate with previously established pluripotency markers and was sufficient to allow for the simple purification and culture of hPSCs after magnetic bead separation. Most importantly, molarity competition with soluble carbohydrates allowed for the elution of the biotin-lectin-bead complex from the cell surface, thus allowing for unaltered cells to be obtained and used in subsequent experiments [8].
In summary, lectin binding and glycan profiling can be used for the identification, selection and separation of pluripotent cells that can then be used in a variety of stem cell applications. Of importance is the suitability of such an approach for the separation of mixed cell populations as well as the possibility for multi-species studies. Furthermore, the fact that lectins can be easily removed from the cell surface by simple competition and elution, make lectin-based sorting strategies a reliable alternative for living cell applications. Considering that lectin profiles are able to discriminate between metabolic cell states, cell activation and even transformation, lectin profiling and cell separation could be used for the study of intermediate populations during both, the reprogramming and the differentiation processes [8-10]. Altogether, lectin-based profiling and sorting brings an unmatched tool not only for the separation of hPSCs prior to transplantation or disease modeling studies but also for more fundamental studies aiming to decipher the molecular mechanisms governing stem cell fate.

\section{References}

1 Takahashi K, Yamanaka S. Induction of pluripotent stem cells from mouse embryonic and adult fibroblast cultures by defined factors. Cell 2006; 126:663676.

2 Inoue H, Yamanaka S. The use of induced pluripotent stem cells in drug de- velopment. Clin Pharmacol Ther 2011; 89:655-661.

3 Sun N, Longaker MT, Wu JC. Human iPS cell-based therapy: considerations before clinical applications. Cell Cycle 2010; 9:880-885.

4 Andrews PW, Banting G, Damjanov I, Arnaud D, Avner P. Three monoclonal antibodies defining distinct differentiation antigens associated with different high molecular weight polypeptides on the surface of human embryonal carcinoma cells. Hybridoma 1984; 3:347361.

5 Zhong B, Watts KL, Gori JL, et al. Safeguarding nonhuman primate iPS cells with suicide genes. Mol Ther 2011; 19:1667-1675.

6 Kuno A, Uchiyama N, Koseki-Kuno $\mathrm{S}$, et al. Evanescent-field fluorescenceassisted lectin microarray: a new strategy for glycan profiling. Nat Methods 2005; 2:851-856.

7 Budnik BA, Lee RS, Steen JA. Global methods for protein glycosylation analysis by mass spectrometry. Biochim Biophys Acta 2006; 1764:1870-1880.

8 Wang YC, Nakagawa M, Garitaonandia I, et al. Specific lectin biomarkers for isolation of human pluripotent stem cells identified through array-based glycomic analysis. Cell Res 2011; 21:1551-1563.

9 Toyoda M, Yamazaki-Inoue M, Itakura $\mathrm{Y}$, et al. Lectin microarray analysis of pluripotent and multipotent stem cells. Genes Cells 2011; 16:1-11.

10 Tateno $\mathrm{H}$, Toyota M, Saito S, et al. Glycome diagnosis of human induced pluripotent stem cells using lectin microarray. J Biol Chem 2011; 286:2034520353. 